03

\title{
Условия коалесценции объемных нанопузырьков
}

\author{
() С.И. Кошоридзе
}

Институт прикладной механики РАН, Москва, Россия

E-mail: koshoridze-semen@yandex.ru

Поступило в Редакцию 25 марта 2020 г.

В окончательной редакции 25 марта 2020 г.

Принято к публикации 13 апреля 2020г.

Рассматривается коалесценция двух объемных нанопузырьков с существенно различными радиусами. Изучение данного вопроса имеет практическое значение, так как коалесценция - процесс нежелательный (нанопузыри, укрупняясь, теряют свои уникальные свойства). В упрощенной модели заряженных нанопузырьков на основе энергетического подхода показано, что в отличие от случая поверхностных нанопузырей для объемных нанопузырей при определенных значениях плотности поверхностного заряда, концентрации и отношения радиусов коалесценция может иметь место.

Ключевые слова: объемный нанопузырь, коалесценция, электростатическое давление, созревание Оствальда.

DOI: 10.21883/PJTF.2020.14.49662.18307

Нанопузырьки (НП) находят широкое применение в различных химических, физических и биологических процессах; следовательно, важно увеличить время жизни НП, предотвращая их растворение и коалесценцию [1-3].

Как известно [1], поверхностные НП стабильны относительно диффузионного растворения при выполнении двух обязательных условий: 1) вода должна быть перенасыщена газом, находящимся внутри НП; 2) граница раздела твердой, газообразной и жидкой фаз должна быть закреплена (так называемый пиннинг). Если на подложке рядом находятся НП с различными радиусами кривизны, то при выполнении отмеченных выше условий не будет наблюдаться „оствальдовское созревание“ диффузионное растворение малых НП и рост больших НП [2]. Математическое моделирование показывает [3], что при этих условиях подавляется также коалесценция, в частности поглощение малых поверхностных НП большими.

Для объемных НП ситуация неопределенная. Нет ясности в том, какой физический механизм защищает их от быстрого диффузионного растворения. В [4] было высказано предположение, что устойчивость объемных НП связана с их поверхностным зарядом. Действительно, в средах с $\mathrm{pH}>4.5$ нанопузыри обладают отрицательным зарядом, создаваемым предположительно ионами $\mathrm{OH}^{-}$, и соответственно отрицательным электрокинетическим потенциалом от 25 до $40 \mathrm{mV}$ [5,6]. Заряд, создавая „отрицательное“ растягивающее давление, частично или полностью компенсирует сжимающее НП лапласовское давление и в результате уменьшает скорость растворения НП в воде. Что касается „оствальдовского созревания“, а также коалесценции мелких и крупных НП, то в литературе этот вопрос ни теоретически, ни экспериментально пока не рассматривался.

В настоящей работе на основе простой модели заряженных НП [7-10] исследована коалесценция, а конкрет- но поглощение объемных НП более крупными НП в зависимости от их концентрации $c$ и плотности поверхностного заряда $\sigma$, а также относительных размеров НП.

Рассмотрим объемные НП в воде. Меньшие НП с радиусом $R$ обозначим как $S$, а более крупные с радиусом $n R(n>1)-$ как $B$. Давление газа внутри НП в состоянии динамического равновесия равняется

$$
p_{S}=\frac{2 \alpha}{R}+p_{a}-\frac{\sigma^{2}}{2 \varepsilon \varepsilon_{0}}, \quad p_{B}=\frac{2 \alpha}{n R}+p_{a}-\frac{\sigma^{2}}{2 \varepsilon \varepsilon_{0}},
$$

для $S$ и $B$ соответственно. Здесь $\alpha-$ поверхностное натяжение воды, $\varepsilon_{0}$ - электрическая постоянная, $\varepsilon-$ диэлектрическая проницаемость воды, $\sigma$ - плотность поверхностного заряда НП, $p_{a}$ - атмосферное давление над поверхностью воды. Третье слагаемое в правой части каждого из уравнений (1) - так называемое электростатическое давление [4,7-10].

$$
\begin{gathered}
\sigma_{S}^{e q}=\sqrt{2 \varepsilon \varepsilon_{0}\left(p_{a}-p_{p}\right)+\frac{4 \alpha \varepsilon \varepsilon_{0}}{R}} \approx \sqrt{\frac{4 \alpha \varepsilon \varepsilon_{0}}{R}}, \\
\sigma_{B}^{e q}=\sqrt{2 \varepsilon \varepsilon_{0}\left(p_{a}-p_{p}\right)+\frac{4 \alpha \varepsilon \varepsilon_{0}}{n R}} \approx \sqrt{\frac{4 \alpha \varepsilon \varepsilon_{0}}{n R}}
\end{gathered}
$$

- равновесные значения поверхностного заряда для $S$ и $B$ (видно, что $\left.\sigma_{B}^{e q}<\sigma_{S}^{e q}\right)$, при которых давление в НП равно парциальному давлению этого газа в атмосфере $p_{p}$ [4] и газ внутри НП находится в диффузионном равновесии с тем же газом, растворенным в воде. При переходе на приблизительные равенства в (2) учтено очевидное, справедливое для НП условие

$$
p_{a}-p_{p} \ll \frac{2 \alpha}{n R} .
$$

Если поверхностные заряды меньше равновесных $\left(\sigma<\sigma_{B}^{e q}<\sigma_{S}^{e q}\right)$, НП растворяются в воде. Максимально 
возможное значение поверхностного заряда НП, согласно (1), равно

$$
\sigma_{\max }=\sqrt{2 p_{a} \varepsilon \varepsilon_{0}+\frac{4 \alpha \varepsilon \varepsilon_{0}}{n R}} \approx \sqrt{\frac{4 \alpha \varepsilon \varepsilon_{0}}{n R}} .
$$

Из (2), (4) следует, что равновесное $\sigma_{B}^{e q}$ и максимально возможное $\sigma_{\max }$ значения плотности заряда для $B$ фактически совпадают друг с другом. С другой стороны, равновесное значение заряда для $S$ удовлетворяет неравенству $\sigma_{\max }<\sigma_{S}^{e q}$. Это означает, что $S$ всегда будет растворяться в воде.

Суммарная тепловая энергия $S$ и $B$ в приближении идеального газа вычисляется по формуле

$$
E_{t h}=\frac{p_{S} 4 \pi R^{3}}{3(\gamma-1)}+\frac{p_{B} 4 \pi(n R)^{3}}{3(\gamma-1)}
$$

где $\gamma$ - показатель адиабаты газа, находящегося внутри НП.

$$
E_{s}=4 \pi \alpha R^{2}+4 \pi \alpha(n R)^{2}
$$

- суммарная поверхностная энергия,

$$
E_{e}=\frac{2 \pi R^{3} \sigma^{2}}{\varepsilon \varepsilon_{0}}+\frac{2 \pi(n R)^{3} \sigma^{2}}{\varepsilon \varepsilon_{0}}
$$

- суммарная собственная электростатическая энергия.

Пусть $c$ - общая концентрация всех НП. Для простоты будем считать, что $S$ и $B$ имеют одинаковые концентрации, равные $c / 2$, и учтем взаимодействие только ближайших соседей. Координационное число (количество ближайших соседей) для простоты примем равным 6 (как в простой кубической решетке). Тогда

$$
E_{C}=3 \frac{\sqrt[3]{c}\left(4 \pi R^{2} \sigma\right)^{2}}{4 \pi \varepsilon \varepsilon_{0}}+5 \frac{\sqrt[3]{c}\left(4 \pi R^{2} \sigma\right)^{2} n^{2}}{4 \pi \varepsilon \varepsilon_{0}}+3 \frac{\sqrt[3]{c}\left(4 \pi n^{2} R^{2} \sigma\right)^{2}}{4 \pi \varepsilon \varepsilon_{0}}
$$

- сумма кулоновской энергии взаимодействия между $S$ и $S$ (первое слагаемое), $S$ и $B$ (второе слагаемое), $B$ и $B$ (третье слагаемое). В (8) учтено, что у $S$ есть три ближайших $S$-соседа, у $B$ тоже три ближайших $B$-соседа. У $B$ есть три ближайших $S$-соседа, а у $S-$ три ближайших $B$-соседа. Поскольку одна связь $B-S$ учтена дважды, во втором слагаемом стоит коэффициент 5, а не 6.

Рассмотрим процесс слияния $B$ и $S$. Для простоты будем считать коалесценцию изотермическим процессом, что для идеального газа равносильно условию

$$
E_{t h}=\text { const. }
$$

Для определения радиуса $R_{c}$ образовавшегося НП исходя из (1), (5), (9) можно написать

$$
\begin{aligned}
\left(\frac{2 \alpha}{R}\right. & \left.+p_{a}-\frac{\sigma^{2}}{2 \varepsilon \varepsilon_{0}}\right) R^{3}+\left(\frac{2 \alpha}{n R}+p_{a}-\frac{\sigma^{2}}{2 \varepsilon \varepsilon_{0}}\right) n^{3} R^{3} \\
& =\left(\frac{2 \alpha}{R_{c}}+p_{a}-\frac{\sigma_{c}^{2}}{2 \varepsilon \varepsilon_{0}}\right) R_{c}^{3} .
\end{aligned}
$$

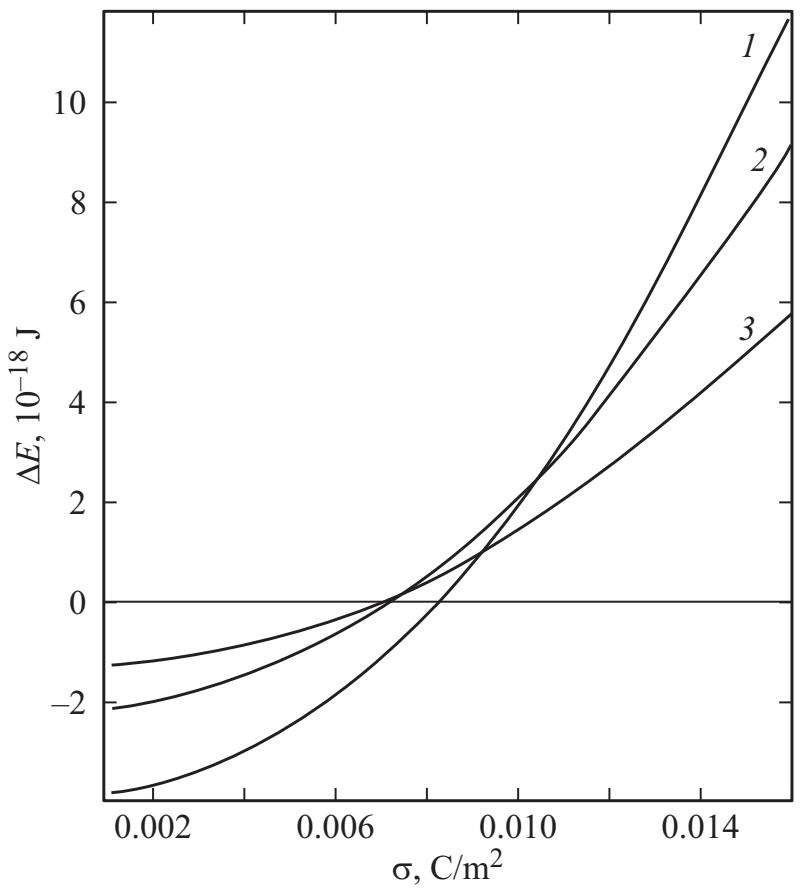

Рис. 1. Зависимость изменения полной энергии при коалесценции $S$ и $B(\Delta E)$ от плотности поверхностного заряда $(\sigma)$ при различных значениях отношения радиусов $B$ и $S$ (n). $n=5(1), 3(2)$ и 2 (3). Расчетные данные: $R=10 \mathrm{~nm}$, $c=10^{15} \mathrm{~m}^{-3}, p_{a}=10^{5} \mathrm{~Pa}, \alpha=0.072 \mathrm{~J} / \mathrm{m}^{2}, \varepsilon=81$.

Здесь

$$
\sigma_{c}=\sigma\left(1+n^{2}\right)\left(\frac{R}{R_{c}}\right)^{2}
$$

- плотность поверхностного заряда образовавшегося НП. Эта величина должна быть меньше максимально возможного значения (ср. с (4)):

$$
\sigma_{c}<\sqrt{2 p_{a} \varepsilon \varepsilon_{0}+\frac{4 \alpha \varepsilon \varepsilon_{0}}{n R_{c}}} .
$$

При сохранении общего объема газа в процессе коалесценции $S$ и $B$ радиус образовавшегося НП был бы равен $R_{c 0}=R \sqrt[3]{1+n^{3}}$. Расчеты показывают, что $R_{c}>R_{c 0}$, т. е. объем образовавшегося НП больше суммы объемов $S$ и $B$.

Для изменения поверхностной $\Delta E_{s}$, электростатической $\Delta E_{e}$ и кулоновской $\Delta E_{C}$ энергий в процессе коалесценции $S$ и $B$ получаем (изменение тепловой энергии в нашей модели, согласно $\left.(9), \Delta E_{t h}=0\right)$ соответственно

$$
\begin{aligned}
& \Delta E_{s}=4 \pi \alpha\left(R_{c}^{2}-R^{2}-n^{2} R^{2}\right)=4 \pi \alpha R^{2}\left[\left(\frac{R_{c}}{R}\right)^{2}-1-n^{2}\right], \\
& \Delta E_{e}=\frac{2 \pi \sigma^{2}}{\varepsilon \varepsilon_{0}}\left(R_{c}^{3}-R^{3}-n^{3} R^{3}\right)=\frac{2 \pi \sigma^{2} R^{3}}{\varepsilon \varepsilon_{0}}\left[\left(\frac{R_{c}}{R}\right)^{3}-1-n^{3}\right],
\end{aligned}
$$




$$
\begin{aligned}
& \Delta E_{C}=\frac{\sqrt[3]{c / 2}\left(4 \pi R_{c}^{2} \sigma\right)^{2}}{4 \pi \varepsilon \varepsilon_{0}}-3 \frac{\sqrt[3]{c}\left(4 \pi R^{2} \sigma\right)^{2}}{4 \pi \varepsilon \varepsilon_{0}} \\
& -5 \frac{\sqrt[3]{c}\left(4 \pi R^{2} \sigma\right)^{2} n^{2}}{4 \pi \varepsilon \varepsilon_{0}}-3 \frac{\sqrt[3]{c}\left(4 \pi n^{2} R^{2} \sigma\right)^{2}}{4 \pi \varepsilon \varepsilon_{0}} \\
& =\frac{\sqrt[3]{c}\left(4 \pi R^{2} \sigma\right)^{2}}{4 \pi \varepsilon \varepsilon_{0}}\left[\frac{1}{\sqrt[3]{2}}\left(\frac{R_{c}}{R}\right)^{4}-3-5 n^{2}-3 n^{4}\right] .
\end{aligned}
$$

Коалесценция будет энергетически выгодна при отрицательном значении изменения полной энергии $S$ и $B$ после их слияния

$$
\Delta E=\Delta E_{s}+\Delta E_{e}+\Delta E_{C}<0
$$

Результаты расчета величины $\Delta E$ по формулам (13)-(16) представлены на рис. 1,2. Параметры подбирались так, чтобы плотность поверхностного заряда НП, образованного после коалесценции $\sigma_{c}$, удовлетворяла неравенству (12), так как иначе статическое равновесие между сжимающими и „отрицательными“ (растягивающими) давлениями нарушится. Таким образом, обязательным (но недостаточным) условием коалесценции НП является то, что величина $\sigma$, согласно (11), (12), должна быть меньше некоторого критического

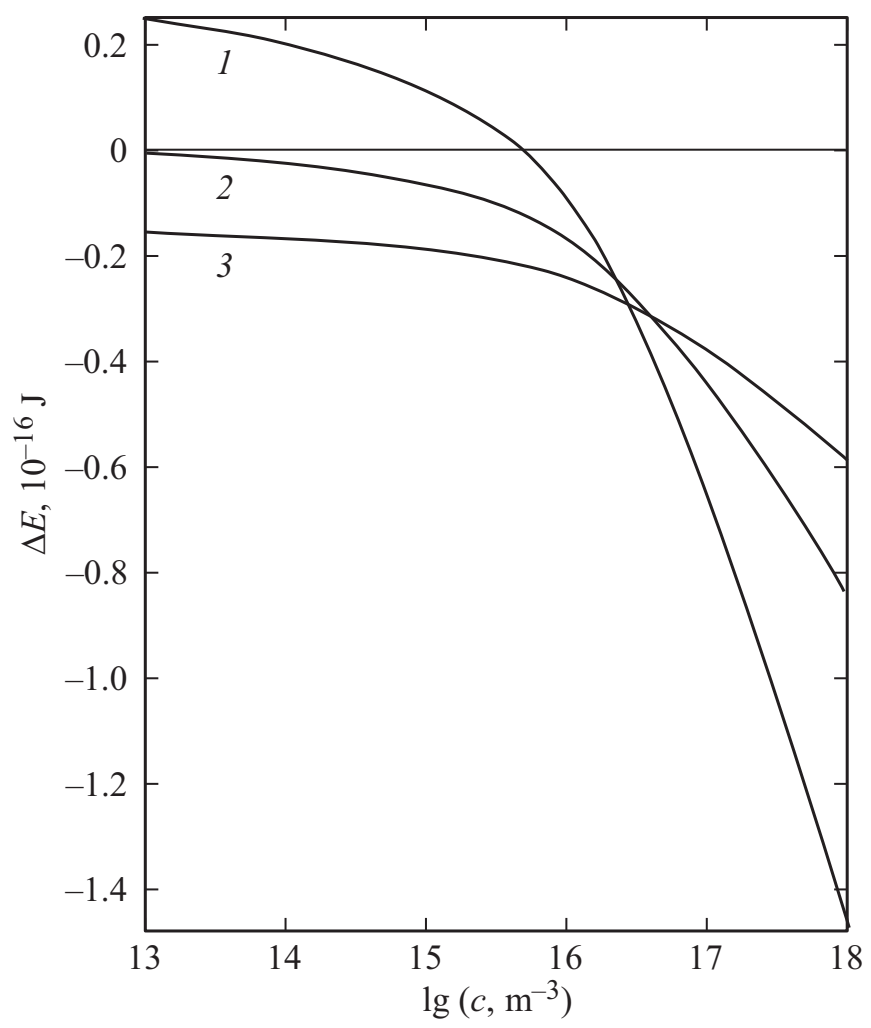

Рис. 2. Зависимость изменения полной энергии при коалесценции $S$ и $B(\Delta E)$ от концентрации НП $(c)$ при различных значениях плотности заряда $(\sigma) . \sigma, \mathrm{C} / \mathrm{m}^{2}: 1-0.010,2-0.007$, 3 - 0.005. Расчетные данные: $n=4, R=20 \mathrm{~nm}$, остальные параметры те же, что на рис. 1. значения $\sigma_{c r}$ :

$$
\sigma<\sigma_{c r} \equiv \frac{\left(\frac{R_{c}}{R}\right)^{2} \sqrt{2 p_{a} \varepsilon \varepsilon_{0}+\frac{4 \alpha \varepsilon \varepsilon_{0}}{n R_{c}}}}{1+n^{2}} .
$$

Итак, согласно нашим расчетам, коалесценции способствуют 1) уменьшение плотности поверхностного заряда $\sigma ; 2)$ рост отношения радиусов $B$ и $S$; 3) увеличение концентрации $c$ НП. Следовательно, при больших $\sigma$ подавляется не только диффузионное растворение НП, но и коалесценция - слияние $B$ и $S$ и в конечном счете укрупнение НП.

Анализ простой модели объемных заряженных НП показал следующее.

1. Коалесценция малых $(S)$ и более крупных $(B)$ объемных НП стимулируется большими значениями отношения их радиусов $n$ и концентраций $c$ и малыми значениями плотности поверхностного заряда $\sigma$.

2. Коалесценция может происходить только в том случае, когда величина $\sigma$ меньше так называемого критического значения $\sigma_{c r}$ (см. (17)). Это обязательное, но недостаточное условие протекания коалесценции. При $\sigma_{c r}<\sigma<\sigma_{\max }$ коалесценция принципиально невозможна.

3. Равновесное значение плотности поверхностного заряда для $S$ всегда больше максимально возможного значения заряда: $\sigma_{S}^{e q}>\sigma_{\max }$, т.е. $S$ всегда нестабильны относительно растворения в воде.

4. Тогда как для поверхностных НП коалесценция и „оствальдовское созревание“ маловероятны, в нашей простой модели заряженных объемных НП коалесценция малых и более крупных НП при определенных параметрах возможна.

\section{Конфликт интересов}

Автор заявляет, что у него нет конфликта интересов.

\section{Список литературы}

[1] Dollet B., Lohse D. // Langmuir. 2016. V. 32. P. 11335-11339. https://doi.org/10.1021/acs.langmuir.6b02136

[2] Lohse D., Zhang X. // Phys. Rev. E. 2015. V. 91. P. 031003. https://doi.org/10.1103/PhysRevE.91.031003

[3] Li C., Zhang A-M., Wang S., Cui P. // AIP Adv. 2018. V. 8. P. 015104. https://doi.org/10.1063/1.5013244

[4] Бункин Н.Ф., Бункин Ф.В. // УФН. 2016. Т. 186. № 9. C. 933-952. DOI: 10.3367/UFNr.2016.05.037796

[5] Calgaroto S., Willberg K.Q., Rubio J. // Miner. Eng. 2014. V. 60. P. 33-40. http://doi.org/10.1016/j.mineng.2014.02.002

[6] Chaplin M. Water structure and science. [Электронный ресурс]. Режим доступа: 1sbu.ac.uk/water/water_structure_science.html (2020).

[7] Боменятов Б.В., Кочоридзе С.И., Левин Ю.К. // Изв. вузов. Физика. 2018. Т. 61. № 10. С. 149-155. 
[8] Кошоридзе С.И., Левин Ю.К. // 8-я Всерос. науч. конф. „Механика композиционных материалов и конструкций, сложных и гетерогенных сред“. Сб. трудов. М., 2019. C. 141-144.

[9] Кочоридзе С.И., Левин Ю.К. // 8-я Всерос. науч. конф. „Механика композиционных материалов и конструкций, сложных и гетерогенных сред“. Сб. трудов. М., 2019. C. $145-149$.

[10] Кошоридзе С.И., Левин Ю.К. // 7-я Всерос. науч. конф. „Механика композиционных материалов и конструкций, сложных и гетерогенных сред“. Сб. трудов. М., 2017. C. $237-239$. 